Research Article

\title{
Simultaneous Determination of Methamphetamine and Its Isomer N-Isopropylbenzylamine in Forensic Samples by Using a Modified LC-ESI-MS/MS Method
}

\author{
Yangxu Luo, ${ }^{1}$ Juan Du, ${ }^{1,2}$ Huadi Xiao, ${ }^{1}$ Ling Zheng, ${ }^{1}$ Xuncai Chen $\mathbb{D}^{,},{ }^{2,3}$ Ande Ma $\mathbb{D}^{1,2}$ \\ and Qizhi Luo $\mathbb{D}^{2,3}$ \\ ${ }^{1}$ Hygiene Detection Center, School of Public Health, Southern Medical University, Guangzhou, China \\ ${ }^{2}$ Forensic Science Center, Southern Medical University, Guangzhou, China \\ ${ }^{3}$ Department of Forensic Toxicology, School of Forensic Medicine, Southern Medical University, Guangzhou, China \\ Correspondence should be addressed to Xuncai Chen; xche3815@smu.edu.cn, Ande Ma; mandmy@126.com, \\ and Qizhi Luo; luoqizhi12@126.com
}

Received 17 December 2020; Revised 23 December 2020; Accepted 12 January 2021; Published 3 February 2021

Academic Editor: Hassan Karimi-Maleh

Copyright (C) 2021 Yangxu Luo et al. This is an open access article distributed under the Creative Commons Attribution License, which permits unrestricted use, distribution, and reproduction in any medium, provided the original work is properly cited.

\begin{abstract}
Accurate identification and quantification of methamphetamine (MA) and its related substances are essential for the investigation and fair trial of drug offenses. In this study, a modified LC-ESI-MS/MS method for the simultaneous determination of MA and its isomer $\mathrm{N}$-isopropylbenzylamine (N-IBA) in forensic samples was developed and validated. Optimum chromatographic separation of the target analytes was achieved on an Agilent Poroshell 120 SB-C18 column $(4.6 \times 100 \mathrm{~mm}, 2.7 \mu \mathrm{m})$ at $40^{\circ} \mathrm{C}$ with isocratic elution at the flow rate of $0.40 \mathrm{~mL} / \mathrm{min}$. The mobile phase was acetonitrile and $20 \mathrm{mM}$ ammonium acetate solution containing $0.1 \%$ formic acid $(80: 20, v / v)$. Positive ESI-MS/MS detection was performed in multiple reaction monitoring (MRM) mode to identify and quantify the target analytes. Method validation showed excellent linearity in the range of $0.51 \mathrm{ng} / \mathrm{mL} \sim 51 \mathrm{ng} / \mathrm{mL}$ for MA and N-IBA. The low limit of detection (LLOD) and low limit of quantification (LLOQ) reached $0.1 \mathrm{ng} / \mathrm{mL}$ and $0.3 \mathrm{ng} / \mathrm{mL}$ for both analytes. The method showed a satisfactory accuracy with an inter- and intraday-relative error (RE) $<20 \%$, and a precision of inter- and intraday relative standard deviation (RSD) less than 15\%. The validated method was successfully applied in real forensic samples and resulted in the detection of MA and N-IBA in 8 suspected samples in drug cases that only deemed MA positive using our previous routine screening procedure, which avoided the misidentification of N-IBA as MA.
\end{abstract}

\section{Introduction}

Drug abuse has been increasingly becoming one of the most severe social problems all over the world. Among drugs of abuse, methamphetamine (MA) is the second most popular illicit drug worldwide which has been listed as a category I psychotropic substance under strict state control in most countries [1]. According to the United Nations Office on Drugs and Crime (UNODC), MA is one of the most dangerous drugs due to its central nervous excitatory effect, highly addictive, and numerous related disorders [2-4]. MA causes toxicity such as liver, brain neurological, cardiovascular, immune system injury, physical, and mental problems (violence, anxiety, and paranoia) [5-11]. Thus, accurate identifi- cation and quantification of MA in the forensic samples is particularly important for investigations and fair trials of drug crimes [12]. However, an isomer of MA, called Nisopropylbenzylamine (N-IBA), has often been used as the adulterant of MA in drug crimes due to their high similarity in structure (Figure 1), which easily resulted in the misidentification of N-IBA as MA in suspected samples [13]. For example, the forensic science laboratory of the United States Drug Enforcement Administration (DEA) reported several cases of counterfeiting MA hydrochloride with N-IBA hydrochloride from 2007 to 2008 [14]. Since 2011, the material identification center of the Ministry of State Security of the People's Republic of China has also reported several drug cases that N-IBA hydrochloride was mixed with MA 


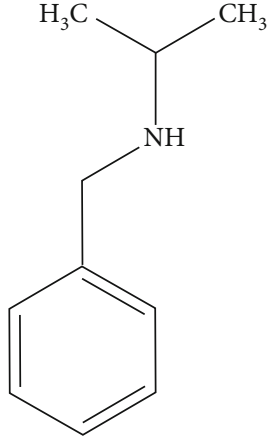

(a)

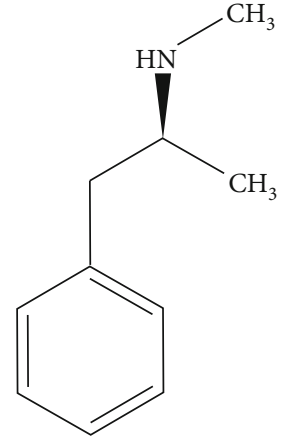

(b)

Figure 1: Chemical structure of (a) N-IBA and (b) MA.

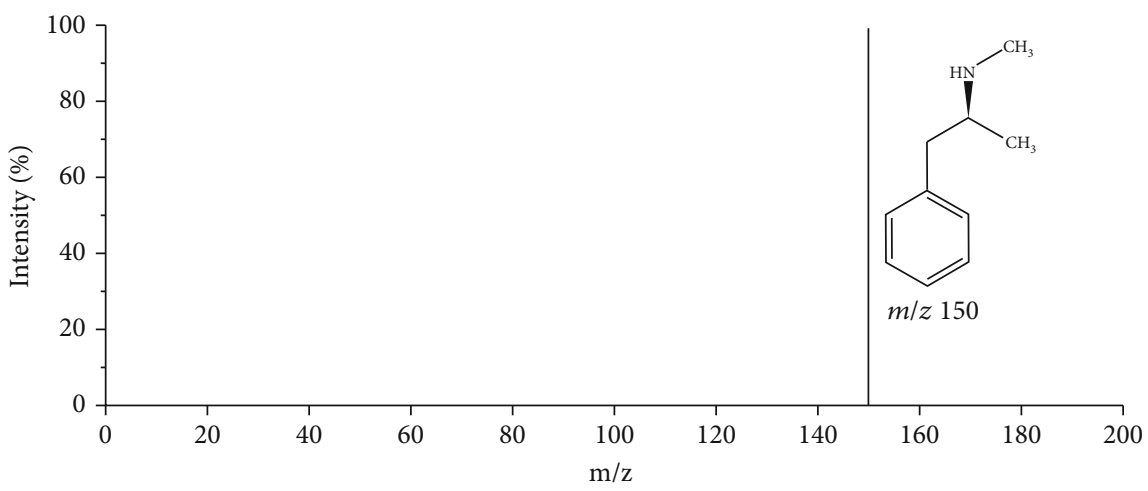

(a)

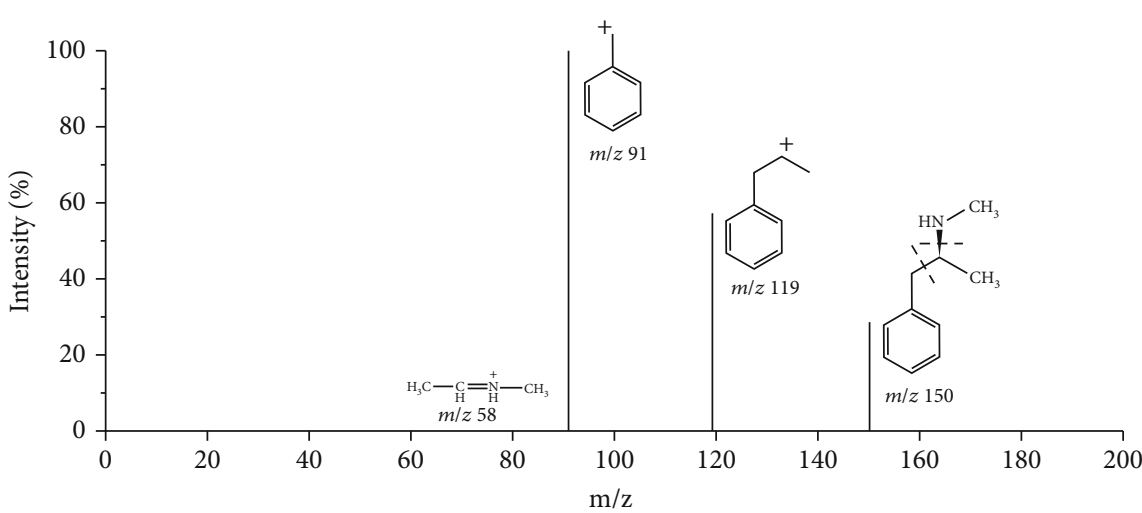

(b)

Figure 2: Mass spectra of methamphetamine (MA). (a) Molecular ion spectrum by (a) mass spectrum by ESI-MS (molecular ion $[\mathrm{M}+\mathrm{H}]^{+}=150$ ). (b) Product ion spectrum by ESI-MS/MS (precursor ion $[\mathrm{M}+\mathrm{H}]^{+}=150$, fragment ions $=119 / 91 / 58$ ).

hydrochloride in China [14]. It is worth to mention that NIBA with unknown toxicity is an important organic synthetic raw material commonly used in industrial productions [13, 15], which is not included in the illicit drug category. The misidentification of N-IBA in MA forensic samples may result in false-positive results, which would cause the misleading legal sentence to the suspects [14]. Therefore, it is necessary to establish an effective detection method for the discrimination of MA and I-NBA in suspected drugs.

At present, many researches have mainly focused on the detection of MA [16-23], while the simultaneous determination of MA and its isomer N-IBA in suspected drugs is rarely studied. The commonly used methods including infrared spectroscopy, colloidal gold-based immunoassays, and color tests are not suitable for the discrimination between MA and N-IBA since their poor specificity [24-28]. GC/MS analysis in full scan mode has been used to simultaneously quantify MA and N-IBA in suspected drugs [25, 27, 28]. However, the two compounds were hard to be effectively discriminated by GC/MS when there was a large concentration difference between them. Because the retention times for MA and N-IBA chromatographic separation were very close due to their high similar chemical structure, the compound with high concentration would 


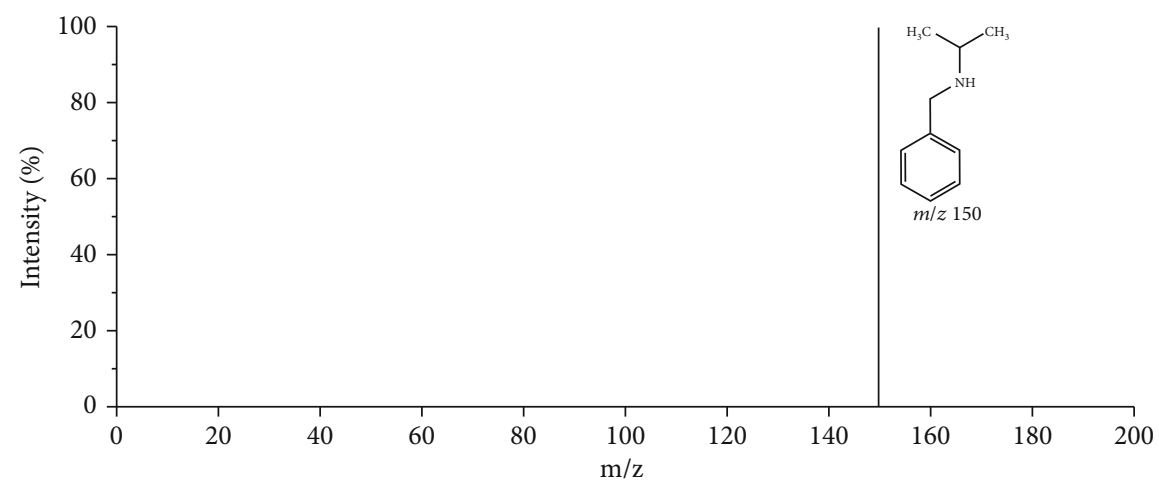

(a)

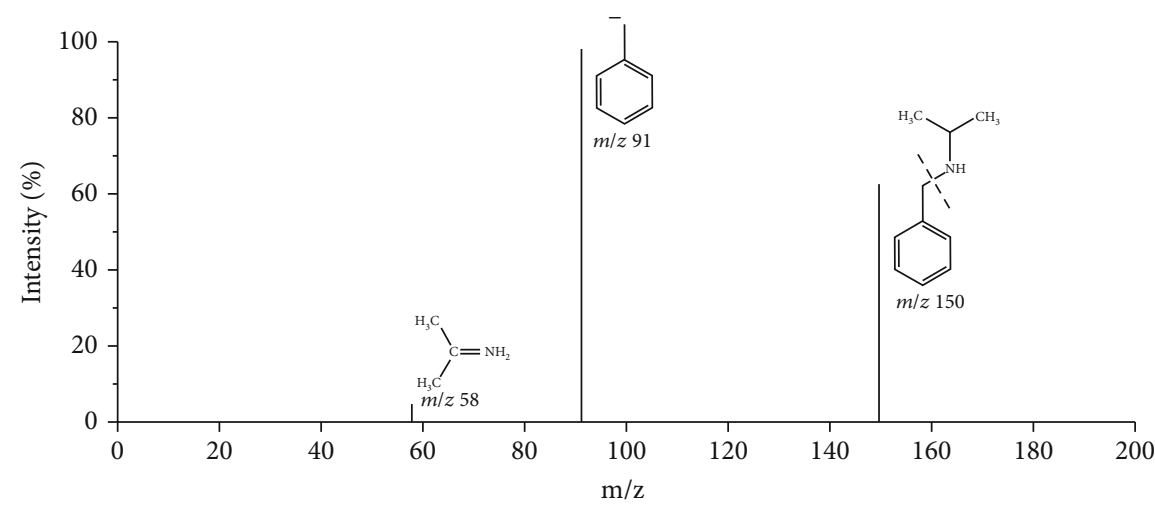

(b)

Figure 3: Mass spectra of N-isopropylbenzylamine (N-IBA). (a) Molecular ion spectrum by (a) mass spectrum by ESI-MS (molecular ion $[\mathrm{M}+\mathrm{H}]^{+}=150$ ). (b) Product ion spectrum by ESI-MS/MS (precursor ion $[\mathrm{M}+\mathrm{H}]^{+}=150$, fragment ions $=91 / 58$ ).

interfere with another one with low concentration as the two compounds yield similar ion fragments for detection [25]. Liquid chromatography-mass spectrometry (LCMS/MS) has fully proved itself as a powerful tool for detecting and confirming the presence of drugs in complex matrices [29, 30]. In consideration of the highly similar ion fragments between MA and N-IBA, the commonly used LC-MS/MS is insufficient to separate the MA from $\mathrm{N}$-IBA. Hence, to develop a high efficient chromatographic separation technique in LC-MS/MS is highly desirable for the simultaneous determination of $\mathrm{MA}$ and its isomer $\mathrm{N}$ IBA.

In this work, a modified LC-ESI-MS/MS method conducted with positive electrospray ionization (ESI) in multiple reaction monitoring (MRM) mode was subsequently developed and validated to discriminate MA and N-IBA in forensic science [24]. Optimum chromatographic separation of the target analytes was achieved on an Agilent Poroshell $120 \mathrm{SB}^{-\mathrm{C}_{18}}$ column $(4.6 \times 100 \mathrm{~mm}, 2.7 \mu \mathrm{m})$ at $40^{\circ} \mathrm{C}$ with isocratic elution at the flow rate of $0.40 \mathrm{~mL} / \mathrm{min}$. The method was successfully applied to determine the MA and N-IBA with satisfactory selectivity, sensitivity, accuracy, and repeatability. In addition, 8 suspected samples in drug cases deemed MA positive were screened by using this new method, and the N-IBA was seized in all samples, illustrating the ubiquity of adulteration of N-IBA to MA.

\section{Materials and Methods}

2.1. Chemicals and Reagents. MA (purity 98\%) was purchased from Merck Sigma-Aldrich (Darmstadt, Germany). N-IBA (purity 98\%) was purchased from Energy Chemical (Shanghai, China). Methanol and acetonitrile of HPLC grade were purchased from Merck Millipore (Darmstadt, Germany). Ammonium acetate (purity 99.0\%) was purchased from Aladdin Bio-Chem Technology Co., Ltd. (Shanghai, China). Formic acid of HPLC grade was from Tianjin Kemiou Chemical Reagent Co., Ltd. (Tianjin, China). Ultrapure water $(R>18 \mathrm{M} \Omega / \mathrm{cm})$ was obtained from a Millipore Milli-Q system (Merck Millipore, Darmstadt, Germany).

2.2. Solutions. Individual stock solutions of MA and N-IBA were prepared in methanol at the concentration of $1 \mathrm{mg} / \mathrm{mL}$ and stored at $-20^{\circ} \mathrm{C}$ for up to 6 months. Appropriate concentrations of calibrator and QC working solutions were prepared by diluting the stock solutions with methanol. The mixed calibrator solutions were prepared with concentrations of $0.1,0.2,0.5,1.0,5.0,10.0,20.0$, and $50.0 \mathrm{ng} / \mathrm{mL}$. The QC samples were prepared in methanol at the low, medium, and high concentrations of $2.0 \mathrm{ng} / \mathrm{mL}, 10.0 \mathrm{ng} / \mathrm{mL}$, and $20.0 \mathrm{ng} / \mathrm{mL}$. The calibrator solutions, QC solutions were stored at $4^{\circ} \mathrm{C}$ prior to analysis. 

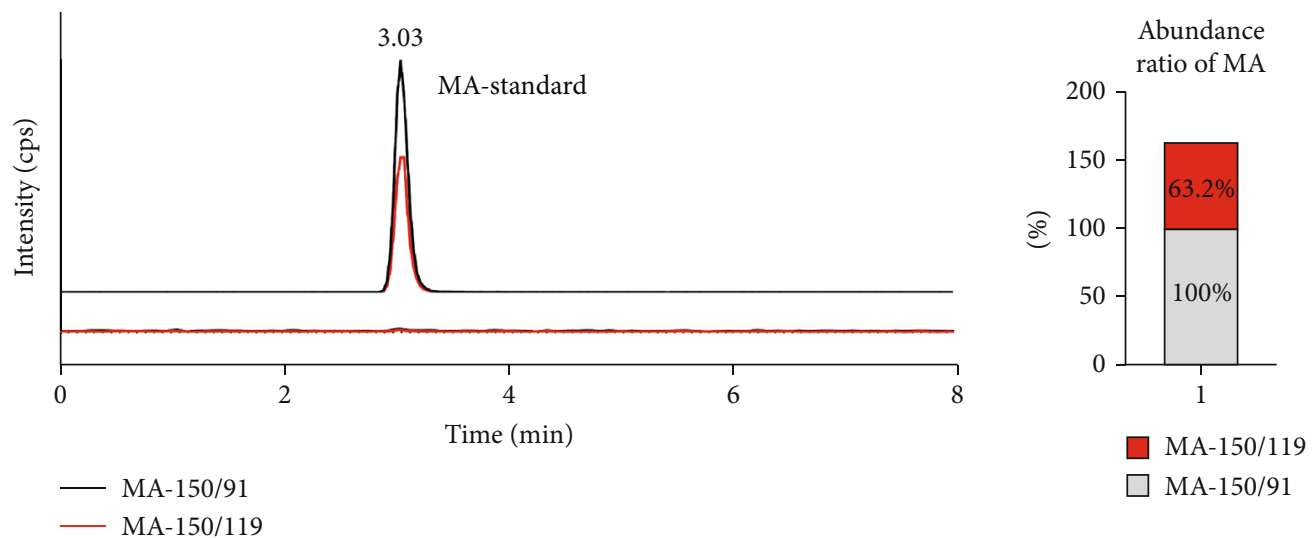

(a)
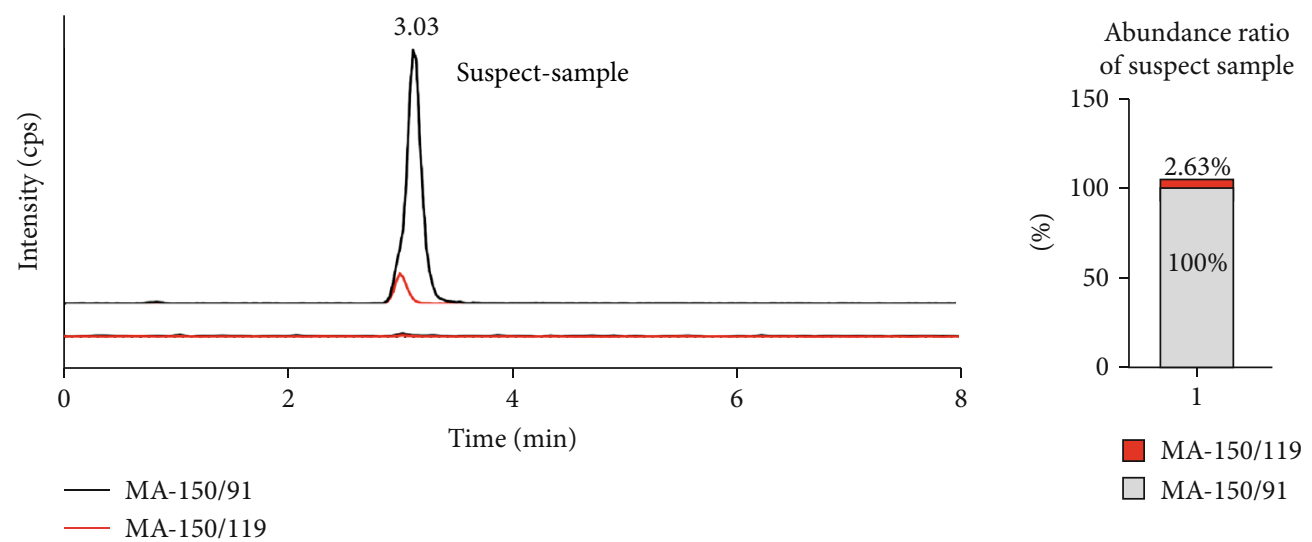

(b)

FIGURE 4: MRM chromatograms for the detection of MA and N-IBA by our routine screening procedures. (a) Upper: detected MA-Standard and the abundance ratio of MA-Standard between $\mathrm{m} / \mathrm{z}$ 150/91and $\mathrm{m} / \mathrm{z} 150 / 119$, lower: blank. (b) Upper: detected suspect sample and the abundance ratio of suspect sample between $\mathrm{m} / \mathrm{z}$ 150/91 and $\mathrm{m} / \mathrm{z}$ 150/119, lower: blank.

2.3. Specimen Preparation. The specimens (white crystalline substances) were obtained from suspected cases of drug production and drug trafficking from 2017 to 2018 in Guangdong province, China. After homogenization, $50 \mathrm{mg}$ of forensic crystal samples was accurately weighed, dissolved in methanol, and gradually diluted to a constant concentration of $1 \mathrm{mg} / \mathrm{mL}, 100 \mu \mathrm{g} / \mathrm{mL}$, and $20 \mathrm{ng} / \mathrm{mL}$. The sample solutions were then filtered by a $0.22 \mu \mathrm{m}$ filter before further analysis.

2.4. Instrumentation. Experiments were performed on a Shimadzu HPLC system (Tokyo, Japan) consisting of two LC20ADvp pumps, a CTO-20ACvp column heater, a SIL-20A autosampler, and a CBM-20A/20Alite controller. The HPLC was interfaced with an AB Sciex API 4000 QTRAP mass spectrometer (Foster City, USA) equipped with an ESI\&Turb spray ionization source. All data were acquired and analyzed using the Analyst software (Version 1.5.2, AB Sciex, Foster City, USA).

\subsection{LC-ESI-MS/MS Conditions}

2.5.1. Routine Screening Procedures. The separation was performed on an RESREK Allure PFPP $\mathrm{C}_{18}$ column $(2.1 \times 100$ $\mathrm{mm}, 5 \mu \mathrm{m})$ protected by RESREK Allure PFPP $\mathrm{C}_{18}$ column $(2.1 \times 10 \mathrm{~mm}, 5 \mu \mathrm{m})$ with isocratic elution at $0.40 \mathrm{~mL} / \mathrm{min}$, giving a total run time of $13.5 \mathrm{~min}$. The column temperature is the same as the room temperature. The mobile phase consisted of methyl alcohol (eluent A) and $20 \mathrm{mM}$ ammonium acetate solution containing $0.1 \%$ formic acid (eluent $\mathrm{B}$ ) in a volume ratio of $70 / 30$. The autosampler tray temperature was maintained at $15^{\circ} \mathrm{C}$, and the injection volume was $20 \mu \mathrm{L}$.

The MS conditions were as follows: the ion polarity was positive, the ionspray voltage applied was $5500 \mathrm{~V}$, the source temperature was set at $600^{\circ} \mathrm{C}$, and the ion source gas $1\left(\mathrm{~N}_{2}\right)$, ion source gas2 $\left(\mathrm{N}_{2}\right)$, and curtain gas $\left(\mathrm{N}_{2}\right)$ were set at 65 psi, 65 psi, and 15 psi, respectively. The ion pairs of MA are $150 / 119$ and $150 / 91$.

2.5.2. Modified Detection Method. The separation was performed on an Agilent Poroshell 120 SB-C 18 column $(4.6 \times 100 \mathrm{~mm}, 2.7 \mu \mathrm{m})$ protected by an Idec Health and Science precolumn (filter assay size: $2 \mu \mathrm{m}$ ) with isocratic elution at $0.40 \mathrm{~mL} / \mathrm{min}$, giving a total run time of $6.5 \mathrm{~min}$. The column temperature was $40^{\circ} \mathrm{C}$. The mobile phase consisted of acetonitrile (eluent A) and $20 \mathrm{mM}$ ammonium acetate solution containing $0.1 \%$ formic acid (eluent B) in a volume ratio of $20 / 80$ [14, 19]. The 
autosampler tray temperature was maintained at $15^{\circ} \mathrm{C}$, and the injection volume was $10 \mu \mathrm{L}$.

MS/MS parameters were optimized with standard solutions of each analyte infused into the ESI source at $10 \mu \mathrm{L} / \mathrm{min}$ via a syringe infusion pump. The MS conditions were as follows: the ion polarity was positive, the ionspray voltage applied was $5500 \mathrm{~V}$, the source temperature was set at $600^{\circ} \mathrm{C}$, and the ion source gas $1\left(\mathrm{~N}_{2}\right)$, ion source gas $2\left(\mathrm{~N}_{2}\right)$, and curtain gas $\left(\mathrm{N}_{2}\right)$ were set at $55 \mathrm{psi}, 65 \mathrm{psi}$, and $15 \mathrm{psi}$, respectively. MRM scans were performed with each analyte utilizing one molecular ion and two most predominant fragments; of the two, the most sensitive transition was used as quantifier ions and the other transition as qualifier ions, the ratios of which are indicative of the analyte of interest. The selected MRM transitions, declustering potentials and collision energies for each analyte are given in Supplementary material Table 1.

2.6. Method Validation. This method was validated according to the CFDA drug analytical method validation guidelines with minor modifications [31]. The validation items include selectivity, the calibration curves and their linearity, LLOD and LLOQ, accuracy, precision, and stability.

2.6.1. Selectivity and Discrimination Capability. Method selectivity was tested by comparing the chromatograms obtained from six blank samples free of MA and N-IBA with those from standard solutions. The extracted ion chromatograms at the retention times of the target analytes were examined for interfering peaks.

The discrimination capability of the method was evaluated by analyzing low, medium, and high concentrations of mixed standard solutions of MA and N-IBA. The extracted ion chromatograms were then inspected for unequivocal identification of the two isomers.

2.6.2. Calibration Curves. The calibration curves were constructed by running six concentration levels of standard solutions ranging from $0.5 \mathrm{ng} / \mathrm{mL}$ to $50 \mathrm{ng} / \mathrm{mL}$ in three replicates. Determination coefficients $\left(R^{2}\right)$ were determined by applying a weighted $\left(1 / \mathrm{x}^{2}\right)$ least-squares linear regression. Calibrators and triplicate of QC samples at low, medium, and high concentrations were analyzed daily in each set of specimens.

2.6.3. LLOD and LLOQ. LLOD and LLOQ were evaluated using a triplicate of standard solutions at the lowest calibration concentrations of the target analytes. LLOD was defined as the concentration producing a Gaussian-shaped peak eluting within $\pm 2 \%$ of mean calibration retention time, a minimum signal to noise $(\mathrm{S} / \mathrm{N})$ ratio of $3: 1$; LLOQ was defined as the concentration that met LLOQ criteria and quality control precision and accuracy within $\pm 20 \%$ and had a minimum $\mathrm{S} / \mathrm{N}$ ratio of $10: 1$.

2.6.4. Accuracy and Precision. Intraday precision was evaluated using five replicated of QC solutions at low, medium, and high concentrations $(2.0,10.0$, and $20.0 \mathrm{ng} / \mathrm{mL}$, respectively) on a single day, while interday precision was assessed on three separate days. The precision expressed as RSD,

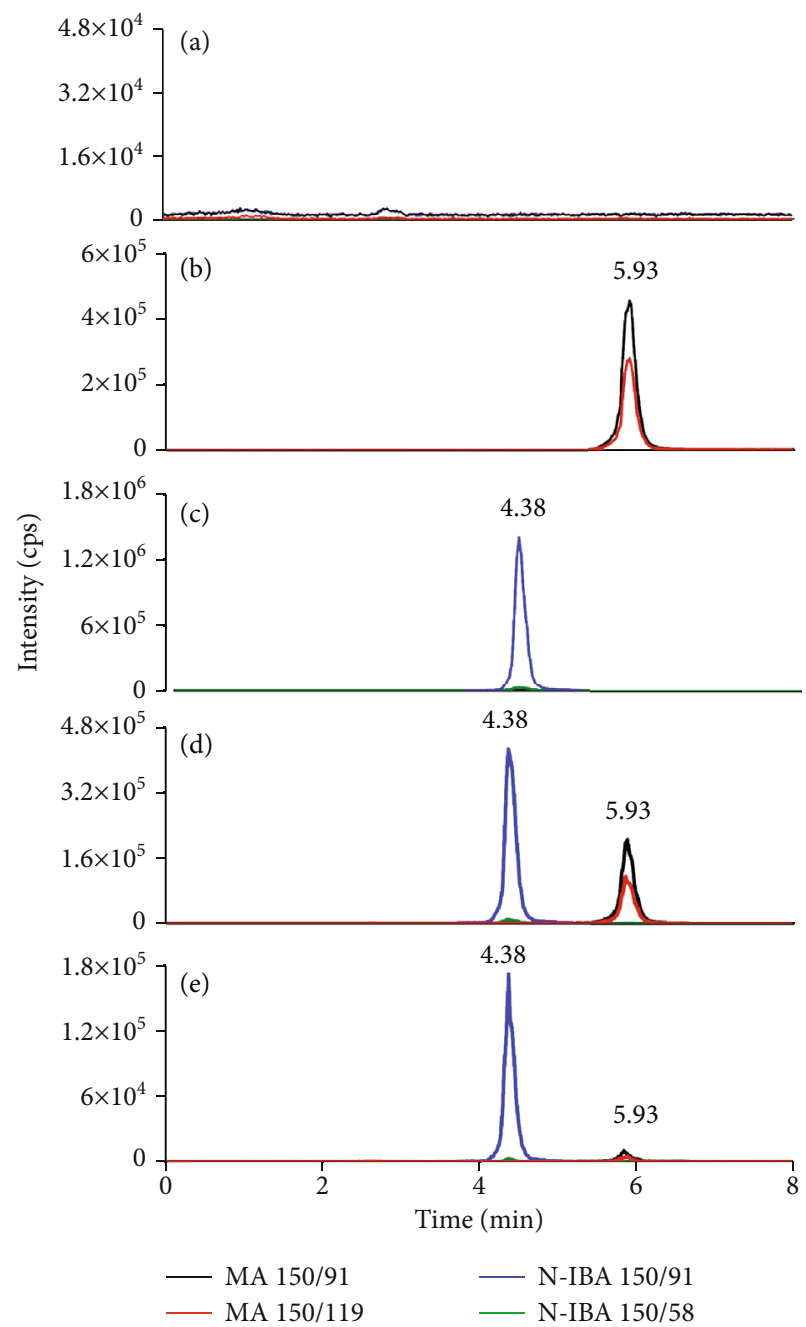

FIGURE 5: MRM chromatograms by LC-ESI-MS for detection of MA and N-IBA. (a) Blank. (b) Standard of MA. (c) Standard of N-IBA. (d) Mix-standard of $\mathrm{MA}$ and N-IBA (N-IBA standard at concentration of $9.14 \mathrm{ng} / \mathrm{mL}$ was mixed with MA standard at concentration of $10.27 \mathrm{ng} / \mathrm{mL}$ at equal volume ratio). (e) Actual forensic sample, SUM-1.

which was calculated as follows:

$$
\mathrm{RSD}=\left[\text { standard deviation }(\mathrm{SD}) / C_{\text {mea }}\right] \times 100 \%
$$

The accuracy was evaluated using triplicate of QC samples solutions at low, medium, and high concentrations; the relative error (RE) was calculated by the mean value of the measured concentrations $\left(C_{\text {mea }}\right)$ from the theoretical concentration $\left(C_{\text {theo }}\right)$.

$$
\mathrm{RE}=C_{\text {mea }} / C_{\text {theo }} \times 100 \%
$$

2.6.5. Stability. Stability of suspected samples, calibrators, and QC samples was tested by reanalyzing them when these samples were kept for three days in an autosampler at $15^{\circ} \mathrm{C}$, one week in room temperature at $20^{\circ} \mathrm{C}$ as well as one month in the freezer at $4^{\circ} \mathrm{C}$. Stability was expressed as the relative 
TABLE 1: Linearity, LLOD, and LLOQ for determining MA and N-IBA.

\begin{tabular}{lccccc}
\hline Compounds & LLOD $(\mathrm{ng} / \mathrm{mL})$ & LLOQ $(\mathrm{ng} / \mathrm{mL})$ & Linear range $(\mathrm{ng} / \mathrm{mL})$ & Equation & $R^{2}$ \\
\hline MA & 0.1 & 0.3 & $0.51 \sim 51.30$ & $\mathrm{y}=3.24 e^{4} x+9.39 e^{4}$ & 0.9959 \\
N-IBA & 0.1 & 0.3 & $0.51 \sim 51.20$ & $\mathrm{y}=8.49 e^{4} x+1.25 e^{4}$ & 0.9973 \\
\hline
\end{tabular}

TABLE 2: The accuracy and precision for quantification of N-IBA and MA.

\begin{tabular}{|c|c|c|c|c|c|}
\hline \multirow{2}{*}{ Compounds } & \multirow{2}{*}{ Nominal concentration $(\mathrm{ng} / \mathrm{mL})$} & \multicolumn{2}{|c|}{ Intraday $(n=5)$} & \multicolumn{2}{|c|}{ Interday $(n=15)$} \\
\hline & & Accuracy (RE, \%) & Precision (RSD, \%) & Accuracy (RE, \%) & Precision (RSD, \%) \\
\hline \multirow{3}{*}{ MA } & 2.05 & 110.1 & 5.4 & 121.9 & 10.0 \\
\hline & 10.27 & 101.8 & 3.5 & 101.0 & 3.6 \\
\hline & 20.53 & 101.1 & 4.0 & 110.0 & 9.9 \\
\hline \multirow{3}{*}{ N-IBA } & 1.83 & 96.2 & 5.1 & 110.7 & 11.6 \\
\hline & 9.14 & 98.5 & 1.7 & 100.1 & 4.3 \\
\hline & 18.28 & 97.2 & 2.7 & 103.6 & 6.4 \\
\hline
\end{tabular}

average deviation (RAD) of the measured concentrations in different time.

\section{Results}

3.1. Development of Confirmatory Method. According to the structural data, N-IBA is an isomer of MA, normally exhibiting similar patterns of collision-induced dissociation (CID) and retention times with MA. Thus, the simultaneous identification for N-IBA and MA was easily confused.

The mass spectra for MA and N-IBA were obtained in full-scan MS and MS/MS mode. The predominant fragments of MA (Figure 2) were $m / z 119\left[\mathrm{M}-\mathrm{CH}_{4} \mathrm{~N}\right]^{+}, m / z 91$ [M$\left.\mathrm{C}_{3} \mathrm{H}_{8} \mathrm{~N}\right]^{+}$, and $m / z 58\left[\mathrm{M}-\mathrm{C}_{7} \mathrm{H}_{7}\right]^{+}$, while the predominant fragments of N-IBA (Figure 3) were $\mathrm{m} / z 91\left[\mathrm{M}-\mathrm{C}_{3} \mathrm{H}_{8} \mathrm{~N}\right]^{+}$ and $m / z 58\left[\mathrm{M}-\mathrm{C}_{7} \mathrm{H}_{7}\right]^{+}$. There are two overlapped fragments $\left(m / z 91\left[\mathrm{M}-\mathrm{C}_{3} \mathrm{H}_{8} \mathrm{~N}\right]^{+}\right.$and $\left.m / z 58\left[\mathrm{M}-\mathrm{C}_{7} \mathrm{H}_{7}\right]^{+}\right)$between MA and N-IBA, besides the specific fragments at $m / z 119$ [M$\left.\mathrm{CH}_{4} \mathrm{~N}\right]^{+}$for MA. Thus, it is difficult to discriminate whether the N-IBA is adulterated in the MA forensic samples by mass spectra directly, especially when the quantity of the adulterated N-IBA is low. Based on our initial analysis of several suspected samples following our routine screening procedures for drug cases, three predominant fragments of MA were observed in the chromatogram of MA, but the unobservable difference was found in the abundance ratio between $\mathrm{m} / \mathrm{z}$ $150 / 91$ and $m / z 150 / 119$, comparing with the MA standard solution. As the analysis result of MA standard solution shown, the predominant fragments of MA were $\mathrm{m} / \mathrm{z} 91, \mathrm{~m} / \mathrm{z}$ 119 , and $\mathrm{m} / z$ 58, and the abundance ratio between $\mathrm{m} / z$ $150 / 91$ and $m / z$ 150/119 was approximately 1:0.63 (Show as Figure 4(a), supplementary material Table 2). The suspected samples showed the same fragments but the higher abundance ratio $(1: 0.026)$ (show as Figure $4(\mathrm{~b})$ ). To see this, we speculate that the existence of N-IBA in the suspected samples interfered the determination of MA. In order to simultaneously determine the N-IBA and MA in the forensic samples, a chromatographical separation was needed to well separate them, before testing them by mass spectra. Several different columns, mobile phases, and gradients were
TABLE 3: Recoveries and RSDs of MA and N-IBA spiked in a suspected drug sample.

\begin{tabular}{lcccc}
\hline Compound & $\begin{array}{c}\text { Background } \\
(\mathrm{ng} / \mathrm{mL})\end{array}$ & $\begin{array}{c}\text { Sample } \\
\text { Add } \\
(\mathrm{ng} / \mathrm{mL})\end{array}$ & $\begin{array}{c}\text { Recovery } \\
(\%)\end{array}$ & $\begin{array}{c}\text { RSD } \\
(\%)\end{array}$ \\
\hline \multirow{3}{*}{ MA } & & 2.05 & 87.8 & 2.8 \\
& 0.48 & 20.50 & 112.6 & 2.1 \\
& & 51.30 & 112.1 & 1.9 \\
\hline \multirow{2}{*}{ N-IBA } & \multirow{2}{*}{5.28} & 1.83 & 96.7 & 0.5 \\
& & 18.30 & 105.1 & 3.4 \\
& & 51.20 & 108.1 & 0.9 \\
\hline
\end{tabular}

studied, and the isocratic separation using acetonitrile and $20 \mathrm{mM}$ ammonium acetate solution containing $0.1 \%$ formic acid $(20: 80, v / v)$ as mobile phase on an Agilent Poroshell $120 \mathrm{SB}^{-\mathrm{C}_{18}}$ column $(4.6 \times 100 \mathrm{~mm}, 2.7 \mu \mathrm{m})$ showed the best separation effect. Then, the MRM transition $m / z$ 150/91 was selected as the quantitative ion pair for MA and N-IBA, and the predominant fragment $\mathrm{m} / z 119$ of MA and $\mathrm{m} / z 58$ of $\mathrm{N}$ IBA was also utilized for the discrimination between MA and N-IBA. Compared to the reported GC/MS method, the excellent chromatographical separation and a minimum of two transitions in the MS/MS spectra for each analyte provide direct evidence to identify MA and N-IBA. The method was subsequently evaluated in compliance with the CFDA drug analytical method validation guidelines with minor modifications.

\subsection{Method Validation}

3.2.1. Selectivity and Discrimination Capability. Selectivity of the method was examined by comparing chromatograms of individual standard solutions with blank sample. As Figure 5(a) showed that no interfering peaks were observed in the chromatogram of blank sample at the retention times of the target analytes.

The capability of the method to discriminate between MA and N-IBA was evaluated by analyzing individual standard 
TABLE 4: The stability of MA and N-IBA under different conditions $(n=3)$.

\begin{tabular}{|c|c|c|c|c|c|}
\hline Conditions & Samples & Compounds & $\begin{array}{l}\text { Day } 0 \text { concentration } \\
(\text { mean } \pm \mathrm{SD}, \mathrm{ng} / \mathrm{mL})\end{array}$ & $\begin{array}{l}\text { Measured concentration } \\
(\text { mean } \pm \mathrm{SD}, \mathrm{ng} / \mathrm{mL})\end{array}$ & $\begin{array}{l}\text { RAD } \\
(\%)\end{array}$ \\
\hline \multirow{4}{*}{$\begin{array}{l}\text { Autosampler for three days } \\
\left(15^{\circ} \mathrm{C}\right)\end{array}$} & \multirow{2}{*}{ Suspect sample } & MA & $1.08 \pm 0.05$ & $1.15 \pm 0.01$ & 3.0 \\
\hline & & N-IBA & $15.27 \pm 0.12$ & $15.13 \pm 0.15$ & 0.4 \\
\hline & \multirow{2}{*}{$\begin{array}{l}\text { Mixed standard } \\
\text { solution }\end{array}$} & MA & $10.43 \pm 0.23$ & $10.23 \pm 0.06$ & 1.0 \\
\hline & & N-IBA & $9.39 \pm 0.18$ & $9.26 \pm 0.20$ & 0.7 \\
\hline \multirow{4}{*}{$\begin{array}{l}\text { Room temperature for one } \\
\text { week }\left(20^{\circ} \mathrm{C}\right)\end{array}$} & \multirow{2}{*}{ Suspect sample } & MA & $1.45 \pm 0.08$ & $1.45 \pm 0.05$ & 0.1 \\
\hline & & N-IBA & $15.33 \pm 0.12$ & $15.00 \pm 0.10$ & 1.1 \\
\hline & \multirow{2}{*}{$\begin{array}{l}\text { Mixed standard } \\
\text { solution }\end{array}$} & MA & $10.53 \pm 0.32$ & $10.21 \pm 0.24$ & 1.5 \\
\hline & & N-IBA & $10.2 \pm 0.20$ & $9.38 \pm 0.18$ & 4.2 \\
\hline \multirow{4}{*}{ Freezer for 30 days $\left(4^{\circ} \mathrm{C}\right)$} & \multirow{2}{*}{ Suspect sample } & MA & $1.16 \pm 0.04$ & $1.10 \pm 0.10$ & 2.4 \\
\hline & & N-IBA & $15.93+0.21$ & $15.33 \pm 0.15$ & 1.9 \\
\hline & \multirow{2}{*}{$\begin{array}{l}\text { Mixed standard } \\
\text { solution }\end{array}$} & MA & $11.23 \pm 0.125$ & $10.43 \pm 0.31$ & 3.7 \\
\hline & & N-IBA & $9.91 \pm 0.14$ & $9.15 \pm 0.06$ & 4.0 \\
\hline
\end{tabular}

solutions and mixed standard solutions at low $(0.2 \mathrm{ng} / \mathrm{mL})$, medium $(20 \mathrm{ng} / \mathrm{mL})$, and high $(100 \mathrm{ng} / \mathrm{mL})$. This range was chosen based on the LLODs and LLOQs. As Figures 5(b) and 5(c) shown, the retention times of MA and N-IBA were approximate 5.86 and $4.36 \mathrm{~min}$, respectively. We also observed single peaks for each target analyte in the chromatograms of mixed standard solutions and suspected samples (Figures 5(d) and 5(e)), indicating the method with highly satisfactory capability of discrimination. Deserved to be mentioned, the routine screening procedure we had performed for psychotropics and narcotics screening more than a few thousands of samples cannot chromatographically separate MA and N-IBA; both compounds have the same retention time, which may result in misidentification. The routine screening samples that had been deemed MA positive were additionally tested, and some samples were ultimately confirmed as N-IBA or mixture of MA and N-IBA.

3.2.2. Linearity, $L L O D$, and $L L O Q$. Linearity was investigated by calculating the coefficient of determination $\left(R^{2}\right)$ of calibration curve, and along with calibration curve equations, linear ranges, LLOD, and LLOQ of each target analyte are listed in Table 1. The calibration curves are in good linearity in the concentrations of $0.51 \sim 51.30 \mathrm{ng} / \mathrm{mL}$ for $\mathrm{MA}$ and $0.51 \sim 51.20 \mathrm{ng} / \mathrm{mL}$ for N-IBA with an $R^{2}$ above 0.99 . The LLODs and LLOQs were $0.1 \mathrm{ng} / \mathrm{mL}$ and $0.3 \mathrm{ng} / \mathrm{mL}$ for MA and N-IBA.

3.2.3. Accuracy and Precision. Accuracy and precision were evaluated with five replicates of QC samples at low, medium, and high concentrations for each target analyte. The results of accuracy and precision of intraday $(n=5)$ and interday $(n=5)$ are shown in Table 2 . The intra- and interday accuracy ranged from $96.2 \%$ to $110.1 \%$ and $100.1 \%$ to $121.9 \%$. The intra- and interday precision ranged from $1.7 \%$ to $5.4 \%$ and from $3.6 \%$ to $11.6 \%$. These results demonstrated that the modified LC-ESI-MS/MS method was reproducible and
TABLE 5: Contents of MA and N-IBA in the suspected samples.

\begin{tabular}{lccc}
\hline $\begin{array}{l}\text { Sample } \\
\text { ID }\end{array}$ & $\begin{array}{c}\text { Quantity weighed } \\
(\mathrm{mg})\end{array}$ & $\begin{array}{c}\text { Contents of N- } \\
\text { IBA }(\%)\end{array}$ & $\begin{array}{c}\text { Contents of } \\
\text { MA }(\%)\end{array}$ \\
\hline SMU-1 & 54.7 & 80.4 & 7.31 \\
SMU-2 & 54.0 & 74.5 & 0.21 \\
SMU-3 & 54.7 & 75.4 & Not detected \\
SMU-4 & 54.6 & 80.4 & Not detected \\
SMU-5 & 55.0 & 0.5 & Not detected \\
SMU-6 & 62.0 & 87.2 & 1.45 \\
SMU-7 & 70.1 & 77.3 & 1.80 \\
SMU-8 & 55.0 & 5.2 & 0.55 \\
\hline
\end{tabular}

reliable for simultaneous determination of MA and N-IBA in forensic samples.

3.2.4. Recovery. Recovery was evaluated by adding low, medium, and high concentration levels of mixed standard solutions into known concentration samples. As shown in Table 3, the recovery value was in the range of $87.8-112.6 \%$, and the RSD value was less than $10 \%$.

3.2.5. Stability. Suspected samples and mixed standard samples were used to evaluate the stability of MA and N-IBA under different conditions. As shown in Table 4, the deviation values between two measured concentrations were less than $5 \%$ of RAD, indicating that MA and N-IBA were stable for three days in an autosampler at $15^{\circ} \mathrm{C}$, for one week in room temperature at $20^{\circ} \mathrm{C}$ and one month in the freezer at $4^{\circ} \mathrm{C}$. Matrix effect, recovery, and stability were within the acceptable range, indicating that the LC-MS/MS method was reliable.

3.3. Application to Real Forensic Drug Samples. During 20172018, 8 suspected samples were screened using our routine screening procedure and deemed MA positive, but the 8 
suspected samples showed a quite unusual abundance ratio between $m / z 150 / 91$ and $m / z$ 150/119 (show as Figure 4). Subsequently, the suspected samples were tested with the modified LC-ESI-MS/MS method, and the adulteration of $\mathrm{N}$-IBA in MA forensic samples was confirmed (Table 5). The main components of these samples were N-IBA and a small amount of MA was found in some samples. Afterwards, all suspected samples in drug cases deemed MA positive were routinely analyzed by the modified LC-ESIMS/MS method to prevent misidentification of MA and NIBA.

\section{Conclusions}

According to the 2018 China drug report [32], MA is the most popular and easily accessible substances for drug addicts in China. In China, those suspected of producing, manufacturing, transporting, and possessing MA will face tough sentences. As we know, N-IBA is the positional isomer of MA, and both isomers have the same retention times using the previous routine screening procedure, which means that the identification of both isomers was easily confused. Since $\mathrm{N}$-IBA is legal to use, misidentification of N-IBA for MA may result in serious consequences.

In the present work, a highly efficient modified LC-ESIMS/MS method was developed and validated for the simultaneous determination of MA and N-IBA in forensic samples. The method exhibited excellent selectivity, linearity, accuracy, precision, and stability with a significantly lower LLOD and LLOQ than those in previous studies. When the method was applied to identify MA and N-IBA in several suspected samples in forensic cases, the MA and N-IBA were well separated and quantified. This work could offer new improvements for the forensic routine analysis of MA and N-IBA in suspected drug samples for clinical and forensic laboratories.

\section{Data Availability}

All data generated or analyzed during this study are included within the article.

\section{Conflicts of Interest}

The authors declare that they have no conflicts of interest.

\section{Authors' Contributions}

Yangxu Luo performed the conceptualization; Yangxu Luo, Juan Du, and Xuncai Chen performed the data curation; Yangxu Luo and Qizhi Luo performed the formal analysis; Juan Du and Ande Ma performed the investigation; Juan Du, Huadi Xiao, and Ling Zheng performed the methodology; Ande Ma and Qizhi Luo gather the resources; Ande Ma performed the validation; Yangxu Luo performed the visualization; Yangxu Luo wrote the original draft; Xuncai Chen and Qizhi Luo wrote, reviewed, and edited the manuscript. Yangxu Luo and Juan Du contributed equally to this work.

\section{Acknowledgments}

This work was supported by the Southern Medical University (grant no: G620522046)

\section{Supplementary Materials}

The following are available online at http://www.mdpi.com/ $\mathrm{xxx} / \mathrm{s} 1$. Table S1: modified detection method of multiple reaction monitoring parameters for determining $\mathrm{MA}$ and N-IBA. Table S2: abundance ratio of MA-Standard and suspect sample. (Supplementary Materials)

\section{References}

[1] P. Boshears, M. Boeri, and L. Harbry, "Addiction and sociality: perspectives from methamphetamine users in suburban USA," Addiction Research \& Theory, vol. 19, no. 4, pp. 289-301, 2011.

[2] J. Ma, X. J. Sun, R. J. Wang et al., "Profile of psychiatric symptoms in methamphetamine users in China: greater risk of psychiatric symptoms with a longer duration of use," Psychiatry Research, vol. 262, pp. 184-192, 2018.

[3] C. McGregor, M. Srisurapanont, J. Jittiwutikarn, S. Laobhripatr, T. Wongtan, and J. M. White, "The nature, time course and severity of methamphetamine withdrawal," Addiction, vol. 100, no. 9, pp. 1320-1329, 2005.

[4] S. R. Radfar and R. A. Rawson, "Current research on methamphetamine: epidemiology, medical and psychiatric effects, treatment, and harm reduction efforts," Addiction and Health, vol. 6, no. 3-4, pp. 146-154, 2014.

[5] Y. Cheng, C. K. Tung, A. K. K. Chung et al., "Screening of pulmonary hypertension in methamphetamine abusers (SOPHMA): rationale and design of a multicentre, crosssectional study," BMJ Open, vol. 9, no. 8, article e027193, 2019.

[6] L. S. Seiden and K. E. Sabol, "Methamphetamine and methylenedioxymethamphetamine neurotoxicity: possible mechanisms of cell destruction," NIDA Research Monograph, vol. 163, pp. 251-276, 1996.

[7] L. Sun, Y. Lian, J. Ding et al., "The role of chaperone-mediated autophagy in neurotoxicity induced by alpha-synuclein after methamphetamine exposure," Brain and Behavior: A Cognitive Neuroscience Perspective, vol. 9, article e01352, 2019.

[8] A. Urbina and K. Jones, "Crystal methamphetamine, its analogues, and HIV infection: medical and psychiatric aspects of a new epidemic," Clinical Infectious Diseases, vol. 38, no. 6, pp. 890-894, 2004.

[9] R. McKetin, A. Voce, R. Burns, and M. Shanahan, "Healthrelated quality of life among people who use methamphetamine," Drug and Alcohol Review, vol. 38, no. 5, pp. 503$509,2019$.

[10] D. C. Vidot, J. A. Manuzak, N. R. Klatt et al., "Brief report: hazardous cannabis use and monocyte activation among methamphetamine users with treated HIV infection," JAIDS Journal of Acquired Immune Deficiency Syndromes, vol. 81, no. 3, pp. 361-364, 2019.

[11] K. Merchant, C. Schammel, and J. Fulcher, "Acute methamphetamine-induced hepatic and pancreatic ischemia," The American Journal of Forensic Medicine and Pathology, vol. 40, no. 3, pp. 285-288, 2019. 
[12] C. Dobkin and N. Nicosia, "The war on drugs: methamphetamine, public health, and crime," The American Economic Review, vol. 99, no. 1, pp. 324-349, 2009.

[13] D. Poppendieck, G. Morrison, and R. Corsi, "Desorption of a methamphetamine surrogate from wallboard under remediation conditions," Atmospheric Environment, vol. 106, pp. 477-484, 2015.

[14] J. Li and H. Liao, "Identification of $\mathrm{N}$-isopropyl-benzylamine in vitro and urine," Forensic Science and Technology, vol. 44, pp. 30-33, 2019.

[15] W. L. Xiong, S. Q. Zhang, C. M. Ji, L. Li, Z. H. Sun, and C. Song, "Temperature-induced reversible isostructural phase transition in _N-isopropylbenzylammonium trifluoromethanesulfonate," Inorganic Chemistry Communications, vol. 41, pp. 79-83, 2014.

[16] S. B. Sachs and F. Woo, "A detailed mechanistic fragmentation analysis of methamphetamine and select regioisomers by GC/MS," Journal of Forensic Sciences, vol. 52, no. 2, pp. 308319, 2007.

[17] A. Oztunc, A. Onal, and S. E. Toker, "Detection of methamphetamine, methylenedioxymethamphetamine, and 3,4methylenedioxy-N-ethylamphetamine in spiked plasma by HPLC and TLC," Journal of AOAC International, vol. 93, pp. 556-561, 2007.

[18] H. T. Andas, A. Enger, A. M. Oiestad et al., "Extended detection of amphetamine and methamphetamine in oral fluid," Therapeutic Drug Monitoring, vol. 38, no. 1, pp. 114-119, 2016.

[19] Y. Zhang, L. Li, Q. Wang et al., "Simultaneous determination of metabolic and elemental markers in methamphetamineinduced hepatic injury to rats using LC-MS/MS and ICPMS," Analytical and Bioanalytical Chemistry, vol. 411, no. 15, pp. 3361-3372, 2019.

[20] M. Di Rago, M. Chu, L. N. Rodda, E. Jenkins, A. Kotsos, and D. Gerostamoulos, "Ultra-rapid targeted analysis of 40 drugs of abuse in oral fluid by LC-MS/MS using carbon-13 isotopes of methamphetamine and MDMA to reduce detector saturation," Analytical and Bioanalytical Chemistry, vol. 48, pp. 3737-3749, 2016.

[21] T. Wang, B. Shen, Y. Shi, P. Xiang, and Z. Yu, "Chiral separation and determination of R/S-methamphetamine and its metabolite R/S-amphetamine in urine using LC-MS/MS," Forensic Science International, vol. 246, pp. 72-78, 2015.

[22] N. Nuntawong, P. Eiamchai, W. Somrang et al., "Detection of methamphetamine $* * / * *$ amphetamine in human urine based on surface $* *-* *$ enhanced Raman spectroscopy and acidulation treatments," Sensors and Actuators B: Chemical, vol. 239, pp. 139-146, 2017.

[23] H. Segawa, Y. T. Iwata, T. Yamamuro et al., "Simultaneous chiral impurity analysis of methamphetamine and its precursors by supercritical fluid chromatography-tandem mass spectrometry," Forensic Toxicology, vol. 37, no. 1, pp. 145-153, 2019.

[24] Q. Tan, Z. Zhang, and H. Bai, "Determination of Nisopropylaniline in workplace air by high-performance liquid chromatography," Zhonghua lao dong wei sheng zhi ye bing $z a$ zhi $=$ Zhonghua laodong weisheng zhiyebing zazhi $=$ Chinese journal of industrial hygiene and occupational diseases, vol. 33, no. 4, pp. 294-296, 2015.

[25] J. Xuan, X. Pan, X. Liu, H. Yan, and Y. Zhang, "Gas chromatography for determination of $\mathrm{N}$-isopropylaniline in work- place atmosphere," Zhonghua Lao Dong Wei Sheng Zhi Ye Bing Za Zhi, vol. 33, no. 2, pp. 143-145, 2015.

[26] R. M. Correia, E. Domingos, F. Tosato et al., "Portable near infrared spectroscopy applied to abuse drugs and medicine analyses," Analytical Methods, vol. 10, no. 6, pp. 593-603, 2018.

[27] K. Sugie, D. Kurakami, M. Akutsu, and K. Saito, "Rapid detection of tert-butoxycarbonyl-methamphetamine by direct analysis in real time time-of-flight mass spectrometry," Forensic Toxicology, vol. 36, no. 2, pp. 261-269, 2018.

[28] J. Welter, M. R. Meyer, E. Wolf, W. Weinmann, P. Kavanagh, and H. H. Maurer, "2-methiopropamine, a thiophene analogue of methamphetamine: studies on its metabolism and detectability in the rat and human using GC-MS and LC(HR)-MS techniques," Analytical and Bioanalytical Chemistry, vol. 405, no. 10, pp. 3125-3135, 2013.

[29] A. Ptak, A. El Tahchy, F. Dupire et al., "LCMS and GCMS for the screening of alkaloids in natural and in vitro extracts of Leucojum aestivum," Journal of Natural Products, vol. 72, no. 1, pp. 142-147, 2009.

[30] T. K. Sinha, R. Maiti, B. Adhikari, and S. K. Ray, "Volatile aroma sensing performance of high quality pristine graphene, obtained via a facile exfoliation technique," Materials Today: Proceedings, vol. 5, no. 3, pp. 9888-9891, 2018.

[31] China Food and Drug Administration (CFDA), "Drug analytical method validation guidelines," 2015, http://samr.cfda.gov.cn/ d i r e c t o r y / w e b/W S $01 /$ i ma ge s / u $6 \mathrm{Rp} 9 \mathrm{Kpzu}$ y s Gv9j WxrfWzva 3 vbeo 0 e $n$ WpLy8yvXWuLW81K3U8i5wZGY=.pdf.

[32] The Ministry of Public Security of the People's Republic of China, "Report on China's drug situation 2018," 2018, http:// www.mps.gov.cn/n6557558/c6535096/content.html. 